\title{
CT-Guided Transparietal Biopsies: Experience of the Radiology Department of the Mohammed VI University Hospital of Marrakech

\author{
Y. El Badri ${ }^{*}$, F.Boukis ${ }^{2}$, K.Elatiqi ${ }^{1}$, H.Rais ${ }^{2}$, Y. Msougar ${ }^{3}$, L. Amrou ${ }^{4}$, B. Boutakioute ${ }^{1}$, M. Ouali Idrissi ${ }^{1}$, N. Cherif Idrissi Ganouni ${ }^{1}$
}

\author{
${ }^{1}$ Radiology Department, Arrazi Hospital, Mohamed VI University Hospital, Cadi Ayyad University, Marrakech, Morocco \\ ${ }^{2}$ Pathology Department, Arrazi Hospital, Mohamed VI University Hospital, Cadi Ayyad University, Marrakech, Morocco \\ ${ }^{3}$ Thoracic Surgery Department, Arrazi Hospital, Mohamed VI University hospital, Cadi Ayyad University, Marrakech, Morocco \\ ${ }^{4}$ Pneumology Department, Arrazi Hospital, Mohamed VI University hospital, Cadi Ayyad University, Marrakech, Morocco
}

The thoracic masses represent a frequent clinical situation of various etiologies. The recent development of new medical imaging techniques, but the confirmation remains histological. Through this three-year retrospective study, we will discuss the diagnostic value of CT-guided transthoracic biopsy in the diagnosis of thoracic lesions. During this period, 334 CT-guided biopsies were performed for suspicion of thoracic tumors, including 259 men and 75 women. The age was ranged between 16 years and 91 years with an average of 56,7 years. 136 patients underwent a bronchoscopy with an inconclusive biopsy. The number of cores sampled varied between 1 and 10 cores. The histological types found were dominated by squamous cell carcinoma $(32,3 \%)$ and adenocarcinoma $(23,9 \%)$, followed by metastases $(9,9 \%)$. The sample did not allow a conclusive pathological analysis in 7 cases. The complications were dominated by alveolar haemorrhage found in 19 cases, followed by pneumothorax / pneumo mediastinum found in 18 cases. CT-guided biopsy is an alternative to invasive surgical biopsy and cytology for the diagnosis of thoracic tumors. It requires a close collaboration between the clinician, the radiologist and the pathologist to discuss its indication, in order to reduce the number of unnecessary biopsies.

Keywords: Thoracic mass, trans parietal biopsy, CT-guided.

Copyright $(\mathcal{C} 2021$ The Author(s): This is an open-access article distributed under the terms of the Creative Commons Attribution 4.0 International License (CC BY-NC 4.0) which permits unrestricted use, distribution, and reproduction in any medium for non-commercial use provided the original author and source are credited.

\section{INTRODUCTION}

Multiple tumor, infectious and inflammatory pathologies can cause a chest mass. Therefor, when a thoracic mass (bronchial, pleural, mediastinal or parietal) is discovered, only histology confirms the etiology. Several methods can be used for obtaining a histological diagnosis: distal biopsies during bronchial fibroscopy (for lung lesions) or ultrasound endoscopy, radioguided trans thoracic biopsy, thoracoscopy or thoracotomy [1]. Radio-guided trans thoracic lung biopsy is currently an essential method for characterizing lung lesions due to its high sensitivity and specificity, as well as its good correlation with thoracotomy results. Initially performed under fluoroscopy, this technique had high complication rates. Currently, with computed tomography (CT) guidance and mastery of this technique, the complication rate has dropped significantly [2]. Our work aims to discuss the value of CT-guided transthoracic biopsy for the diagnosis of chest masses and to try to assess the risk factors correlated with the occurrence of complications.

\section{PATIENTS AND METHODS Patients}

We retrospectively collected all the patients to whom a CT-guided trans thoracic biopsy was performed for a thoracic tumor with anatomopathological confirmation. The study was carried out over five years from January 2015 until the end of December 2019. The pre-biopsy consultation was mandatory to study the file and validate the indication. A hemostasis assessment was required before the procedure (PT> 50\% and platelet count> $50,000)$.

\section{Procedure of the biopsy}

All biopsies were performed by one of the four senior radiologists of the department with the assistance of a junior radiologist.

Preparation of the patient: the patient must be eupneic, informed of the course of the procedure, its 
complications and their possible management in order to obtain informed consent.

Materiel: All biopsies were performed on a 64-strip helical CT (Siemens). We used an automatic $18 \mathrm{G}$ trocar with a $17 \mathrm{G}$ coaxial guide for all patients.

Procedure: The patient is placed on the CT table to the best of his comfort and according to the chosen approach (anterior, posterior or lateral), avoiding fissures, vessels and areas of tumor necrosis. The lateral position on the affected side may be an interesting alternative in order to leave good ventilation on the healthy side and to minimize the risk of complications. $5 \mathrm{~mm}$ contiguous sections without injection of contrast are acquired on the region of interest and skin marking (radiopaque needle) (fig 1) guided by the laser localizer is performed on the "supposed" entry point. The area is cleaned with Betadine and covered with a sterile drape then local anesthesia (Lidocaine $1 \%$ to $2 \%$ ) is done keeping the anesthesia needle in place to verify the entry point by CT sections (fig 2). Sometimes, injection of the contrast is necessary if the lesion presents intimate relations with the vessels and / or hydro-dissection (injection of physiological serum to clear the vessels) particularly for mediastinal lesions (fig 3).

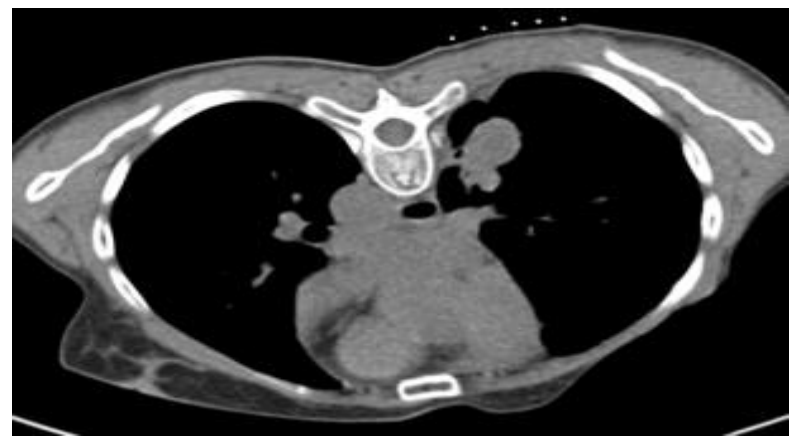

Fig-1: Axial CT image in mediastinal window with radiopaque needles in place, facing the lesion to be biopsied.

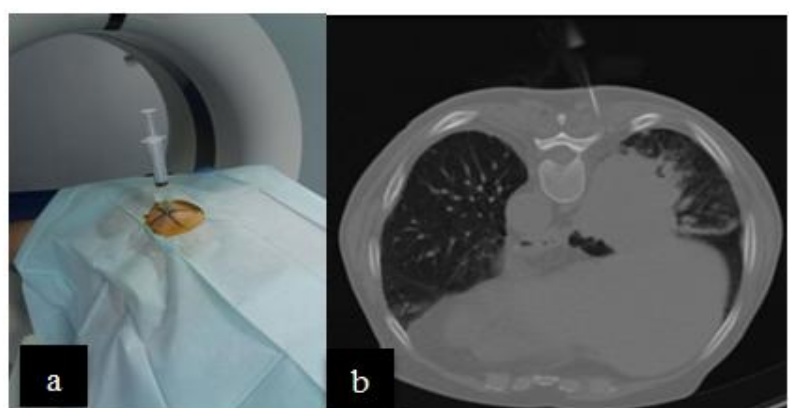

Fig-2 : a) Anesthesia needle kept in place to verify the point of entry. b) Axial CT image in the parenchymal window showing the position of the anesthesia needle to guide the path to be imprinted.

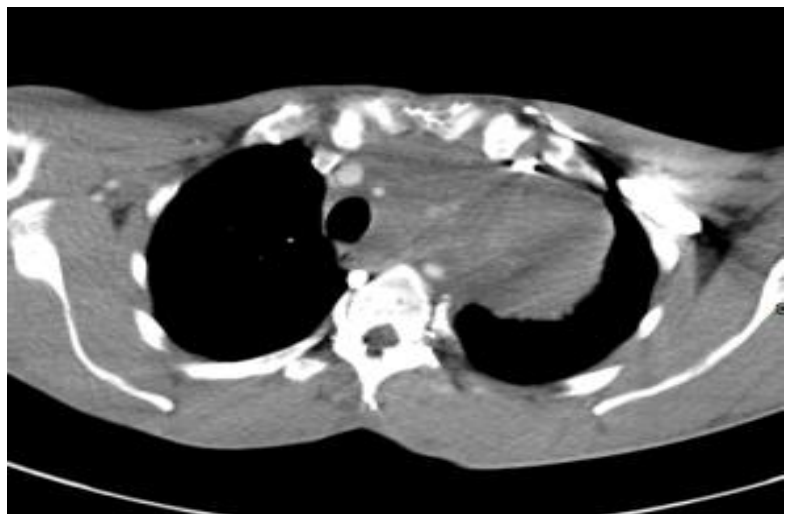

Fig-3: Axial CT image in mediastinal window after the injection of contrast allowing to locate the left internal mammary artery having an intimate contact with the mass to be biopsied.

The guide is then introduced at an angle and depth calculated on the console in a single gesture if possible. After checking its correct position, the internal stylet is withdrawn and the trocar is inserted. The biopsies are taken through the guide, and the carrots are put in formalin and then sent to the pathology laboratory. Finally, rapid removal of the material and compression of the biopsy site. A control acquisition taking the whole thorax is performed. The procedure usually takes 20 to 40 minutes. The patient remains under clinical surveillance, sometimes with a control chest X-ray for two hours after the procedure.

\section{RESULTS}

We collected 334 patients including 259 men and 75 women. The age was ranged from 16 to 91 years old with an average of 56.7 years.

47 patients had diabetes, 16 had cancer and 212 were chronic smokers. The clinical manifestations were dominated by cough in $58.1 \%$ of cases, chest pain in $28.2 \%$, dyspnea in $36 \%$ of cases and haemoptysis in $8.1 \%$ of cases.

Imaging revealed nodular opacities in 64 cases, intraparenchymal mass of more than $3 \mathrm{~cm}$ in 189 cases, excavated opacities in 24 cases, pulmonary condensation in 37 cases, hilar opacity in 41 cases and mediastinal mass in 29 cases. The pulmonary lesions were located in the right lung in $50 \%$ of the case, in the left lung in $33.4 \%$ of the cases and bilateral in $7.8 \%$ of the cases.136 patients underwent a bronchoscopy with prior inconclusive biopsy. 45 patients required an injection of contrast due to the proximity of the lesion to the mediastinal vessels. The number of carrots collected varied between 1 and 6 carrots with an average of 4 carrots. The histological results were distributed according to the location of the lesions (table 1 and fig 4). 
Table-1: Histological results depending on the location of the lesions

\begin{tabular}{|l|l|}
\hline Pleuropulmonary masses & 108 cases $(32,33 \%)$ \\
\hline Squamous cell carcinoma & 80 cases $(23,9 \%)$ \\
\hline Adenocarcinoma & 17 cases $(5 \%)$ \\
\hline Small cell carcinoma & 11 cases $(3,3 \%)$ \\
\hline Large cell carcinoma & 4 cases $(1,2 \%)$ \\
\hline Pulmonary lymphoma & 33 cases $(9,9 \%)$ \\
\hline Metastases & 11 cases $(3,3 \%)$ \\
\hline Pseudotumoral tuberculosis & 32 cases $(9,5 \%)$ \\
\hline Non-specific inflammation & 1 cases $(0,3 \%)$ \\
\hline Pneumoblastoma & 10 cases $(3 \%)$ \\
\hline Mediastinal masses & 18 cases $(5,3 \%)$ \\
\hline Thymoma & 1 case $(0.3 \%)$ \\
\hline Lymphoma & \\
\hline Type T lymphoblastic leukemia &
\end{tabular}

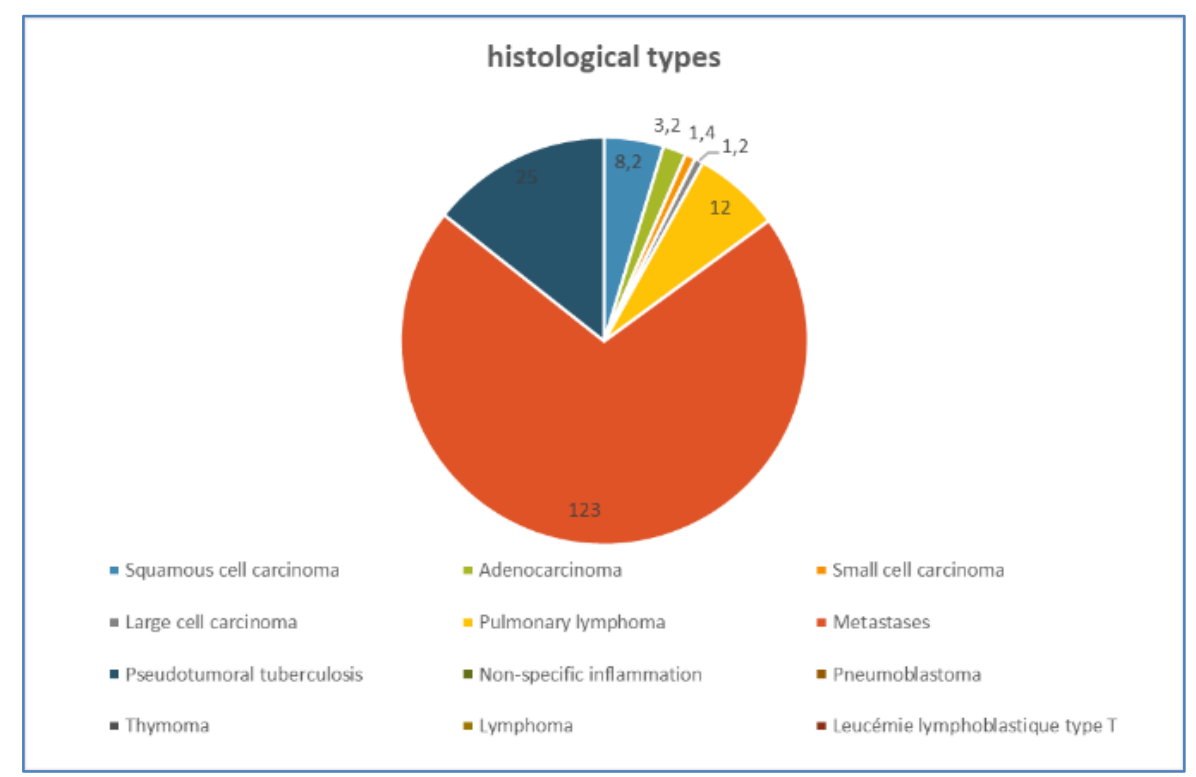

Fig-4: Histological types all locations combined

The sample did not allow a conclusive pathological analysis in 7 cases with a 2nd biopsy (conclusive) performed in 3 cases.

Complications were dominated by alveolar hemorrhage found in 19 cases $(5.5 \%)$ (fig 5), followed by low-abundance pneumothorax found in 18 cases $(5.2 \%$ ) (fig 6). Two cases of post-biopsy death were reported ( $0.6 \%$ of cases) due to ARDS and suffocating pneumothorax.

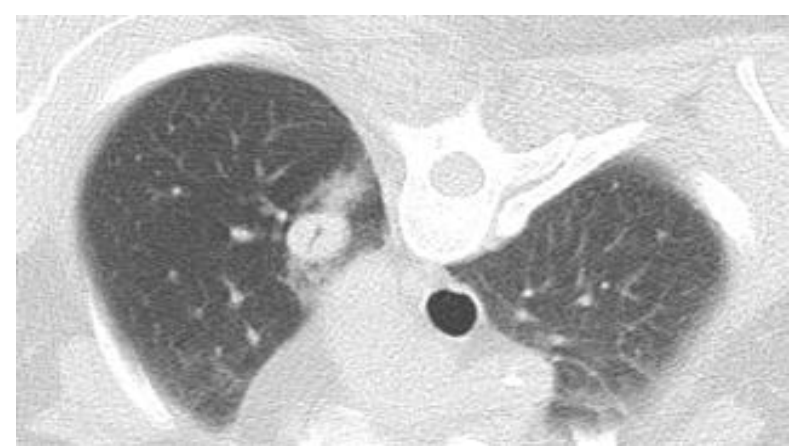

Fig-5: CT image in axial section in parenchymal window showing minimal alveolar hemorrhage after a biopsy.

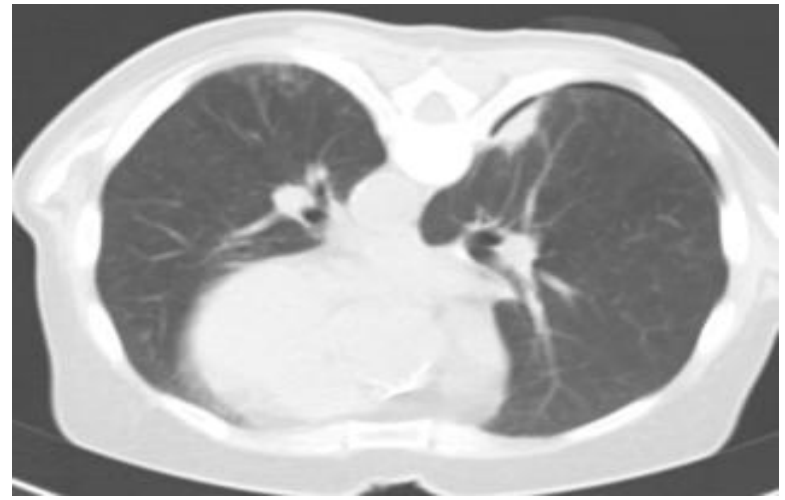

Fig-6: CT image in axial section in parenchymal window showing a right low abundance pneumothorax happening after a CT guided biopsy.

\section{DISCUSSION}

Scanno-guided transthoracic biopsy is an efficient and minimally invasive technique compared to thoracotomy, with sensitivity and specificity varying from $84 \%$ to $97 \%$ and $95 \%$ to $100 \%$ respectively. Introduced for the first time in 1954 by Dutra and Geraci [3] using a coaxial needle or without it and under fluoroscopy, its diagnostic precision has brought 
it great success, despite a high complication rate [1]. Currently, the transthoracic approach has become a routine procedure with the advent of computed tomography and the improvement of biopsy equipments, with significant reduction in the rate of complications. The diagnostic accuracy is estimated between $64 \%$ and $97 \%$ (depending on the size of the lesion) [4]. In our study, the trans thoracic biopsy allowed an accurate diagnosis in $98 \%$ of cases, which could be due to the size of the lesions (the majority being masses of more than $3 \mathrm{~cm}$ in major axis). The different biopsy needles available in the market are divided into two types: the modified Menghini needle and the modified Trucut needle. Theoretically, the first, which makes it possible to take a larger sample corresponding to the caliber of the external cannula, should be better. It is the second type of needle that is the most widely used [5], which is why we have used it in all biopsies.

The CT-guided trans-thoracic biopsy has several indications [6]:

Histology necessary to make a therapeutic decision with failure of bronchoscopy or endobronchial ultrasound. Imaging in favor of malignancy but the patient is inoperable. Appearance or increase of a solitary nodule or of a mass, with negative sample on bronchoscopy, or not accessible lesion.

Multiple nodules in a patient with no history of malignancy, in prolonged remission or known to have multiple primary lesions. Persistent focal infiltrate, single or multiple. Residual nodule after radiotherapy, chemotherapy or thermo-ablation. Sampling of tissues for genomic analysis, therapeutic sensitivity testing, study of tumor markers or hormone dependence, etc. Infectious diseases in healthy or immunocompromised patients resistant to treatment.

On the other hand, it is contraindicated if the patient is dyspneic and / or non-cooperative, in case of hemostasis disorders, severe respiratory failure, significant emphysema, heart failure, pulmonary arterial hypertension, contralateral pneumonectomy, suspicion of hydatid cyst, vascular malformation, meningocele or mediastinal pheochromocytoma. However, no contraindication is absolute, the risk must always be weighed against the expected benefit and we can say that the only absolute contraindication is the absence of an indication [6].

The most common complications of a transthoracic biopsy are pneumothorax and alveolar hemorrhage. The rate of pneumothorax due to biopsy varies a lot between authors, considering recent studies based on large populations, the incidence of post-biopsy pneumothorax is between $19.9 \%$ and $42 \%$ [7]. In our study, the rate of low-abundance pneumothorax was around $5.2 \%$ of cases, mostly negligible but suffocating in only one case.
Factors favoring the occurrence of pneumothorax include: COPD, lack of adherence, elderly or poorly cooperating patient, reduced operator experience, duration of the procedure, diameter and flexibility of the needle, the obliquity of the angle of penetration into the pleura, the multiple repositioning and the number of crossing of the pleura, the depth and diameter of the target, the cavitary character of the lesion, and assistance by mechanical ventilation. Various techniques for reducing the risk of pneumothorax are described, such as the injection of physiological liquid, "blood patching" or the injection of Gelfoam ${ }^{\circledR}$ on the puncture path (intra pulmonary and / or intra pleural); however, these techniques can cause cellular or bacterial dissemination if these substances are injected through the sampling needle [6]. Pulmonary hemorrhage is seen in $4 \%$ to $27 \%$ of CT-guided transthoracic biopsies, the vast majority being limited and non-symptomatic, minor hemoptysis is reported in $4 \%$ of cases. If an internal mammary or intercostal vessel (artery or vein) is involved, hemothorax may occur (approximately $1.5 \%$ of cases). Severe hemoptysis is seen in $0.2 \%$ of cases, and severe hemothorax in $0.09 \%$. The factors influencing the rate of pulmonary hemorrhage are: the small size of the lesion, its depth, the presence of emphysema or pulmonary arterial hypertension [8]. In our series, alveolar hemorrhage was encountered in $5.5 \%$ of cases, no case of hemothorax was described. If there is a small alveolar hemorrhage around the lesion, the procedure can be continued, but immediatly stopped if there is symptomatic hemorrhage with cough and hemopysis. The patient will be placed in lateral decubitus, the biopsied lung in a declining position to avoid flooding of the healthy lung. Bronchial arterial embolization is the treatment of choice for massive hemoptysis, a selective intubation is indicated [6]. Other rare complications can be seen including pulmonary edema, myocardial infarction, pneumonia or bronchospasm; or more rarely coronary or cerebral arterial gas embolism and tumor dissemination along the biopsy path [8]. The overall mortality rate is estimated between $0.015 \%$ and $0.02 \%$ [9]. In our series, two cases of post-biopsy death were reported $(0.6 \%$ of cases), caused by ARDS and suffocating pneumothorax.

\section{CONCLUSION}

The CT-guided trans thoracic biopsy is currently increasingly used. It is a less aggressive technique that has considerably modified the diagnostic behavior in thoracic tumor pathology, while being associated with low complication rates. The development of real-time CT (fluoroscopy-CT, fluoro$\mathrm{CT}$, or CT-continuous) is now widely available and combines the advantages of cross-sectional imaging and real-time control of the procedure. However, the debate continues to revolve around the indications hence the need for close collaboration between the clinician, the radiologist and the pathologist. 


\section{REFERENCES}

1. Namouni M, Decullier E, Réty F, Bendib SE. Performance de la biopsie transthoracique et lésions bénignes. Journal de radiologie. 2011 May 1;92(5):405-11.

2. Smayra T, Braidy C, Menassa-Moussa L. Facteurs de Risque du Pneumothorax et de L'hémorragie Associés à la Biopsie Pulmonaire: Une Expérience Unicentrique. Lebanese Medical Journal. 2012 Jan;103(371):1-0.

3. Dutra FR, Geraci CL. Needle biopsy of the lung. Journal of the American Medical Association. 1954 May 1;155(1):21-4.

4. Birchard KR. Transthoracic needle biopsy. InSeminars in interventional radiology 2011 Mar (Vol. 28, No. 1, p. 87). Thieme Medical Publishers.

5. Yamagami T, Iida S, Kato $\mathrm{T}$, Tanaka $\mathrm{O}$, Toda $\mathrm{S}$, Kato D, Nishimura T. Usefulness of new automated cutting needle for tissue-core biopsy of lung nodules under CT fluoroscopic guidance. Chest. 2003 Jul 1;124(1):147-54.

6. Mills M, Choi J, El-Haddad G, Sweeney J, Biebel B, Robinson L, Antonia S, Kumar A, Kis B.
Retrospective analysis of technical success rate and procedure-related complications of 867 percutaneous CT-guided needle biopsies of lung lesions. Clinical radiology. 2017 Dec 1;72(12):1038-46.

7. Bae K, Ha JY, Jeon KN. Pneumothorax after CTguided transthoracic lung biopsy: A comparison between immediate and delayed occurrence. PloS one. 2020 Aug 24;15(8):e0238107.

8. Heerink WJ, de Bock GH, de Jonge GJ, Groen HJ, Vliegenthart R, Oudkerk M. Complication rates of CT-guided transthoracic lung biopsy: metaanalysis. European radiology. 2017 Jan;27(1):13848.

9. Schulze R, Seebacher G, Enderes B, Kugler G, Fischer JR, Graeter TP. Complications in CTguided, semi-automatic coaxial core biopsy of potentially malignant pulmonary lesions. In RöFoFortschritte auf dem Gebiet der Röntgenstrahlen und der bildgebenden Verfahren. 2015 Aug (Vol. 187, No. 08, pp. 697-702). (C Georg Thieme Verlag KG. 